\title{
Functional impairment in fibrosing alveolitis: relationship to reversible disease on thin section computed tomography
}

\author{
A.U. Wells**+, M.B. Rubens*, R.M. du Bois**, D.M. Hansell*
}

\begin{abstract}
Functional impairment in fibrosing alveolitis: relationship to reversible disease on thin section computed tomography. A.U. Wells, M.B. Rubens, R.M. du Bois, D.M. Hansell. (C)ERS Journals Ltd 1997.

ABSTRACT: Thin-section computed tomography (CT) provides a reproducible method of quantifying global disease extent and can also discriminate between fibrotic disease, with predominance of reticular abnormalities, and reversible inflammatory cell infiltration, shown as ground-glass attenuation.

The aim of this study was to determine whether functional impairment varied according to the presence of ground-glass attenuation on CT, independently of extent of disease on CT, demographic factors, smoking history, therapeutic status, and the type of fibrosing alveolitis (lone cryptogenic fibrosing alveolitis (CFA) versus fibrosing alveolitis associated with systemic sclerosis (FASSc)).

Patients with concurrent emphysema on CT $(n=16)$ and FASSc patients with end-stage pulmonary hypertension $(n=5)$ were excluded. One hundred and eleven patients were studied (CFA, $n=54$; FASSc, $n=57$ ).

The severity of functional impairment did not vary independently with the presence of predominant ground-glass attenuation, mixed appearance and predominant reticulation on CT. In 34 treated patients undergoing serial CT scanning, the severity of functional impairment did not differ independently between patients with and without regression of ground-glass attenuation at follow-up.

We conclude that the severity of functional impairment does not discriminate between inflammatory and fibrotic disease in fibrosing alveolitis, as judged by initial and serial computed tomographic scanning, after adjustment for the morphological extent of disease on computed tomography.

Eur Respir J 1997; 10: 280-285.
\end{abstract}

\begin{abstract}
Dept of *Radiology and **Interstitial Lung Disease Unit, Royal Brompton Hospital London, UK. +Dept of Respiratory Medicine, Green Lane Hospital, Auckland, New Zealand.
\end{abstract}

Correspondence: A.U. Wells Interstitial Lung Disease Unit Royal Brompton Hospital Sydney St

London SW3 6NP

UK

Keywords: Computed tomography fibrosing alveolitis

idiopathic pulmonary fibrosis

pulmonary function testing

Received: February 61996

Accepted after revision October 311996
The histological appearances of fibrosing alveolitis consist of a mixture of inflammatory cell infiltration and interstitial fibrosis [1-3]. It is widely accepted that inflammation precedes and leads to fibrosis; therapies used in fibrosing alveolitis have included anti-inflammatory and immunosuppressive agents [4, 5]. However, it is often difficult to determine how completely inflammation has been suppressed; a poor response to treatment, as judged by plain chest radiographic and physiological criteria, may be indicative of irreversible fibrotic disease but may also reflect residual, potentially reversible inflammatory cell infiltration. The repetition of open lung biopsy is impracticable; appearances on thinsection computed tomography (CT) are a useful guide to the likelihood of reversible inflammatory disease [6, 7], but frequent repetition of CT scanning is constrained by the radiation burden. If a routine pulmonary function variable was found to correlate selectively with the severity of inflammation, it would be an invaluable aid to management. Up to the present time, studies of relationships between functional indices and the severity of inflammation and fibrosis in interstitial lung disease have been based on histological findings at open lung biopsy and have yielded conflicting results [8-11]. How- ever, functional-histopathological correlations suffer from a major flaw: regional disease severity varies greatly in fibrosing alveolitis, and thus the choice of biopsy site introduces major variability.

Characteristic CT appearances discriminate between patients with irreversible fibrotic disease and those with an increased likelihood of significant inflammatory cell infiltration [6, 7]. Moreover, CT provides a reproducible means of quantifying the morphological extent of fibrosing alveolitis [12]. The aim of this study was to determine whether relationships between functional impairment and the extent of fibrosing alveolitis on CT differed between patients with prominent ground-glass attenuation on $\mathrm{CT}$ and those with irreversible reticular disease.

\section{Methods}

\section{Patients}

All patients with cryptogenic fibrosing alveolitis (CFA) or fibrosing alveolitis associated with systemic sclerosis (FASSc) managed by the Interstitial Lung Disease 
Unit of the Royal Brompton Hospital, undergoing concurrent CT of the lungs and pulmonary function tests between June 1, 1985 and October 31, $1989(n=142)$, were eligible. Patients with concurrent emphysema on CT (CFA, n=14; FASSc, n=2), FASSc patients with preterminal pulmonary hypertension, disproportionate to the extent of fibrosing alveolitis $(n=5)$ and patients with incomplete functional data (CFA, $n=8 ; F A S S c, n=2)$ were excluded. The final population consisted of 54 patients with CFA ( 33 males and 21 females; mean \pm sD age $=59 \pm$ 13 yrs) and 57 patients with FASSc (10 males and 47 females; age $48 \pm 12$ yrs). Clinical diagnostic criteria in CFA consisted of: 1) bilateral basal or widespread crackles; 2) a restrictive defect or depression of the transfer factor of the lungs for carbon monoxide (TL,CO; 3 ) $\mathrm{CT}$ abnormalities compatible with a diagnosis of fibrosing alveolitis [13, 14]; and 4) the absence of exposure to a recognized environmental cause of lung fibrosis. In 29 of the 54 patients, the diagnosis of CFA was confirmed at antemortem open-lung biopsy. Patients with FASSc met American Rheumatism Association preliminary criteria for the diagnosis of systemic sclerosis [15] and had evidence of fibrosing alveolitis on CT, confirmed at open-lung biopsy in 34 of the 57 . All patients had been referred for the evaluation of suspected pulmonary complications.

\section{Clinical information}

Demographic details, smoking history, and treatment at the date of CT scanning were extracted from the hospital case-records. Patients smoking at least one cigarette per day for 1 year were classified as "ever-smokers". The total intake of cigarettes was also recorded in "packyears" (one pack of 20 cigarettes per day for one year $=$ one pack-year) in 105 of the 111 patients with a detailed smoking history (CFA, $n=49$; FASSc, $n=56$ ). Treatment was defined as a minimum daily dose of prednisolone of $10 \mathrm{mg}$, with or without cyclophosphamide (CFA, $\mathrm{n}=$ 29; FASSc, n=19). Fifteen FASSc patients were taking penicillamine at an average daily dose of $125-500 \mathrm{mg}$, including 11 patients not receiving steroid or immunosuppressive therapy.

\section{Computed tomography}

CT sections were acquired using an Elscint 2002 scanner (Elscint, Haifa, Israel) with a scanning time of 5.5 s. Interspaced $3 \mathrm{~mm}$ sections were obtained from the lung apices to the lung bases at $10 \mathrm{~mm}$ intervals, with the patients in the supine position, and reconstructed with a high resolution "bone" algorithm. A limited number of sections were performed through the lower zones of the lung with the patient prone (to evaluate the effects of gravity-dependent perfusion). CT scans were evaluated independently by two thoracic radiologists. The extent of fibrosing alveolitis was quantified, as described previously [12]. Each scan was assessed at five levels (the origin of the great vessels, the mid-arch of the aorta, the main carina, the pulmonary venous confluence, and $1 \mathrm{~cm}$ above the right dome of the diaphragm). At each level, a visual assessment was made of the extent of abnormal lung to the nearest 5\%; overall lung involvement was estimated by combining these figures.

Means of the two estimates were used in analysis; this method of determining extent of fibrosing alveolitis has minor interobserver variation [12]. The relative extent of ground-glass attenuation, signifying an increased likelihood of inflammatory cell infiltration $[16,17]$, and a reticular pattern, denoting fibrotic disease [17-19], was categorized as follows: Grade $1=$ ground-glass attenuation predominates; Grade $2=$ both patterns equally extensive ("mixed disease"); Grade 3 = a reticular pattern predominates. This scoring system has a clinically acceptable interobserver variability [12], and is predictive of survival and responsiveness to therapy $[6,7]$.

\section{Indices of lung function}

Pulmonary function tests (expressed as percentages of predicted values) [20] were completed within one month of CT scanning. Forced expiratory volume in one second (FEV1), forced vital capacity (FVC), total lung capacity (TLC), transfer factor of the lungs for carbon monoxide $(T \mathrm{~L}, \mathrm{CO})$ and $T \mathrm{~L}, \mathrm{CO}$ adjusted for alveolar volume (carbon monoxide transfer coefficient $(K \mathrm{CO})$ ) were analysed. Spirometric measurements were performed using a rolling-seal spirometer (Spiroflow, PK Morgan $\mathrm{Ltd}, \mathrm{Kent}, \mathrm{UK})$. Measures of transfer factor $(T \mathrm{~L}, \mathrm{CO}, K \mathrm{CO})$ were performed by the single-breath technique, using Transfer Factor equipment (Model B; PK Morgan Ltd). Arterial blood gas values were measured on air and the alveolar to arterial oxygen tension gradient $\left(\mathrm{A}-\mathrm{a} P, \mathrm{O}_{2}\right)$ was calculated, using the simplified alveolar gas equation [21].

Exercise testing was completed in 81 patients (excluding 30 patients with severe respiratory compromise, or cardiological or rheumatological contraindications to exercise testing). Exercise tests were performed on a treadmill, using the standard Bruce Protocol. Arterial blood gas values were measured at rest and in the last minute of exercise using the end-capillary (ear lobe) technique. A-a $P, \mathrm{O}_{2}$ levels were measured using end-tidal alveolar oxygen values obtained by mass spectrometry. Falls in oxygen saturation between rest and maximal exercise were adjusted for oxygen uptake $\left(V^{\prime} \mathrm{O}_{2}\right)$ achieved as a percentage of maximum predicted $V^{\prime} \mathrm{O}_{2}$, by means of a formula used in the generation of the clinical-radiological-physiological (CRP) score [22]. A logarithmic transformation was performed to correct for positive skewing, for the purposes of parametric analysis $\left(\log \mathrm{D}-\mathrm{O}_{2}\right.$, adj). The physiological component of the CRP score was calculated in 81 patients undergoing exercise testing (CFA, $n=33$; FASSc, $n=48$ ). Indices of lung function were scored as described by WATTERS et al. [22], with the substitution of TLC for thoracic gas volume.

\section{Regression of disease on serial CT}

Change in disease on follow-up CT sections in patients treated between scans was scored as described previously [7]. Analysis was confined to patients in 
whom anatomically comparable sections were available at follow-up. Paired CT sections (of initial and followup scans) were assessed independently by both observers, with the dates of the scans concealed. In patients with more than one anatomically comparable section, all comparable sections were incorporated in a composite assessment. Changes in disease extent were evaluated; for divergent observations, sections were reassessed by both observers and a consensus observation was reached.

\section{Data analysis}

Data are expressed as mean \pm SD. A p-value of less than 0.05 was taken as significant. Group comparisons were made using Student's t-test, one-way analysis of variance (ANOVA) or, when appropriate, by Chi-squared statistics. Analysis was performed using STATA software (Stata data analysis software; Computing Resource Center, Santa Monica, CA, USA). Interobserver variation was expressed as the single determination SD [23] or the kappa coefficient of agreement [24]; a kappa value in excess of 0.40 was taken to indicate fair interobserver agreement [25]. Multivariate linear regression models [26] were constructed to determine whether relationships between functional impairment and the extent of fibrosing alveolitis on CT depended upon which CT pattern predominated, independent of age, sex, smoking history, therapeutic status and the aetiology of fibrosing alveolitis (i.e. CFA versus FASSc). Models were examined for each individual lung function variable, and for the physiological component of the CRP score, with patients categorized as "ever-smokers" or lifetime nonsmokers ("never-smokers"), and, in separate analyses, with smoking history expressed in "pack-years".

\section{Results}

\section{Patients}

Demographic details, smoking histories, extent of disease on CT and indices of lung function are presented in table 1, categorized according to CT appearance. No significant trends were identified across the three CT subgroups; there were approximately equal numbers of patients with CFA and FASSc in each subgroup.

\section{Interobserver variation in scoring extent of disease and predominant pattern on $C T$}

The single determination SD in scoring the extent of fibrosing alveolitis on CT was 5.1\%. There was agreement between observers on the grade for CT appearance in 82 of the 111 cases (74\%), equating to a kappa value of 0.54 (fair to good agreement); consensus agreement on CT grade was reached in the remaining 29 cases. There was agreement between observers as to whether ground-glass attenuation had regressed at follow-up CT in 31 of the 34 cases $(91 \%)$, a kappa value of 0.82 (excellent agreement).
Table 1. - Demographic features, smoking history, extent of disease on CT and indices of lung function, tabulated according to CT appearances

\begin{tabular}{lccc}
\hline & $\begin{array}{c}\text { CT grade 1 } \\
(\mathrm{n}=12)\end{array}$ & $\begin{array}{c}\text { CT grade } 2 \\
(\mathrm{n}=41)\end{array}$ & $\begin{array}{c}\mathrm{CT} \text { grade } 3 \\
(\mathrm{n}=58)\end{array}$ \\
\hline Open lung biopsy $\mathrm{n}$ & 9 & 25 & 29 \\
CFA/FASSc n/n & $6 / 6$ & $17 / 24$ & $31 / 27$ \\
Age yrs & $47(15)$ & $52(13)$ & $56(12)$ \\
Sex M/F & $6 / 6$ & $11 / 30$ & $32 / 26$ \\
Ever-smoked n & 4 & 17 & 27 \\
Abn lung on CT $\%$ & $37(20)$ & $28(19)$ & $32(17)$ \\
FEV1\% pred & $77(24)$ & $76(25)$ & $78(20)$ \\
FVC \% pred & $71(22)$ & $71(26)$ & $72(20)$ \\
TLC \% pred & $70(17)$ & $70(22)$ & $69(18)$ \\
$T \mathrm{~L}, \mathrm{CO} \%$ pred & $46(21)$ & $52(22)$ & $48(18)$ \\
$K \mathrm{CO} \%$ pred & $76(28)$ & $83(19)$ & $80(23)$ \\
$P \mathrm{a}, \mathrm{O}_{2} \mathrm{kPa}$ & $11.0(1.9)$ & $10.9(1.7)$ & $10.6(2.1)$ \\
$\mathrm{Calc} \mathrm{A}-\mathrm{a} P, \mathrm{O}_{2} \mathrm{kPa}$ & $2.9(1.9)$ & $3.1(1.7)$ & $3.3(2.1)$ \\
Meas A-a $P, \mathrm{O}_{2} \mathrm{kPa}$ & $4.0(2.1)$ & $3.4(1.8)$ & $3.1(1.6)$ \\
Log D- $\mathrm{O}_{2}$, adj $^{*}$ & $0.9(0.7)$ & $0.8(0.5)$ & $0.9(0.6)$ \\
\hline
\end{tabular}

CT grade 1=predominant ground-glass attenuation; CT grade $2=$ mixed appearances; CT grade $3=$ predominance of a reticular pattern. Values are presented as mean, and SD in parentheses. *: measured $\mathrm{A}-\mathrm{a} P, \mathrm{O}_{2}$ and $\log \mathrm{D}-\mathrm{O}_{2}$ values were obtained in 81 subjects undergoing maximal exercise testing (CFA, $\mathrm{n}=33$; FASSc, $\mathrm{n}=48$ ). CT: computed tomography; CFA: cryptogenic fibrosing alveolitis; FASSc: fibrosing alveolitis associated with systemic sclerosis; M: male; F: female; Abn: abnormal; FEV1: forced expiratory volume in one second; FVC: forced vital capacity; TLC: total lung capacity; TL,CO: transfer factor of the lung for carbon monoxide; \% pred: percentage of predicted value; KCO: carbon monoxide transfer factor adjusted for alveolar volume (gas transfer coefficient); $\mathrm{Pa}_{\mathrm{a}} \mathrm{O}_{2}$ : arterial oxygen tension; Calc $\mathrm{A}-\mathrm{a} P, \mathrm{O}_{2}$ : alveolar to arterial difference in oxygen tension, calculated using the simplified alveolar gas equation; Meas A-a $P, \mathrm{O}_{2}$ : alveolar to arterial difference in oxygen tension, measured using end-tidal mass spectrometry and end-capillary oxygen sampling; $\log \mathrm{D}-\mathrm{O}_{2}$,adj: fall in oxygen saturation on maximal exercise, adjusted for oxygen uptake $\left(V^{\prime} \mathrm{O}_{2}\right)$ achieved as a percentage of predicted maximum $V^{\prime} \mathrm{O}_{2}$ and transformed logarithmically. No significant group differences were identified.

Functional-morphological relationships analysed according to CT appearance

Multivariate models were constructed to identify independent relationships between CT pattern and the severity of functional impairment (examined as the dependent variable). There was no independent relationship between any individual functional variable and CT pattern, after controlling for the extent of fibrosing alveolitis on CT, demographic factors, smoking histories, therapeutic status, and the type of fibrosing alveolitis. These findings persisted when smoking histories were expressed in "pack-years", and when analysis was confined to 63 patients in whom the diagnosis of fibrosing alveolitis was confirmed by antemortem open-lung biopsy, and to 63 patients who were untreated at initial CT. Functional-morphological relationships for $T \mathrm{~L}, \mathrm{CO}$ and oxygen desaturation on exercise (adjusted for respiratory work) against extent of disease on CT, for CT grades $1-3$, are shown in figures 1 and 2. Subanalyses were performed comparing: 1) patients with CT grade 1 and those with CT grade 3 (excluding patients with mixed 


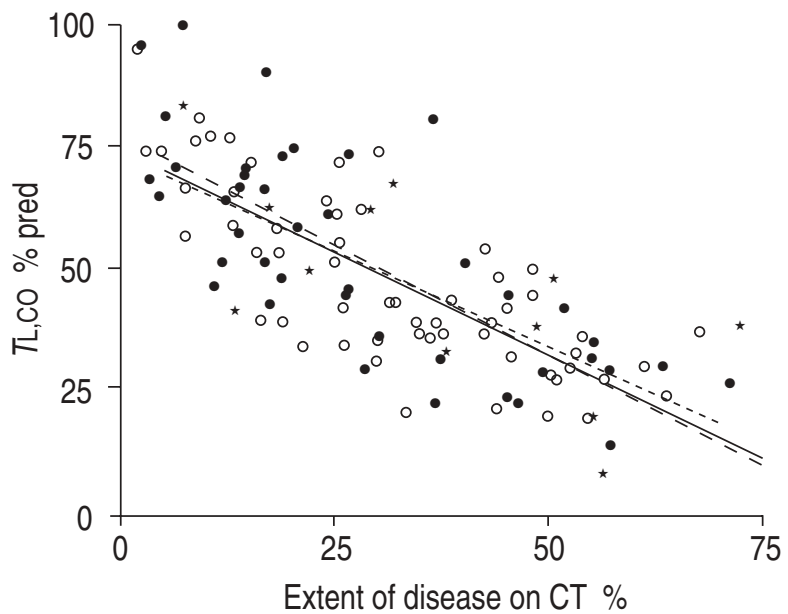

Fig. 1. - Percentage predicted TL,CO in relation to the extent of fibrosing alveolitis on CT in patients with: predominant ground-glass attenuation (CT grade $1 ; \mathrm{r}=-0.68 ; \mathrm{n}=12$ ); mixed appearances (CT grade $2 ; \mathrm{r}=-0.78 ; \mathrm{n}=41)$; and predominance of a reticular pattern (CT grade $3 ; \mathrm{r}=-0.76 ; \mathrm{n}=58)$. Functional-morphological relationships were not influenced by CT appearance. $\star$ and - - - -: CT grade 1 ; $\bullet$ and --- : CT grade 2; 0 and - $\longrightarrow$ CT grade 3 . CT: computed tomography; $T \mathrm{~L}, \mathrm{CO}$ : transfer factor of the lung for carbon monoxide; $\%$ pred: percentage of predicted value.

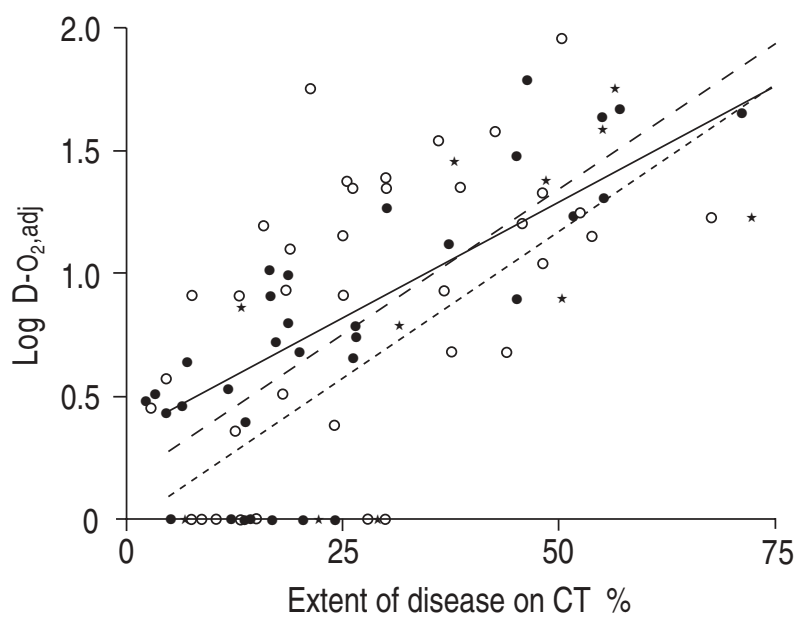

Fig. 2. - Oxygen desaturation on maximal exercise, adjusted for respiratory work and transformed logarithmically $\left(\log \mathrm{D}_{-} \mathrm{O}_{2}, \mathrm{adj}\right)$, in relation to the extent of fibrosing alveolitis on computed tomography (CT) in patients with: predominant ground-glass attenuation (CT grade $1 ; \mathrm{r}=0.73 ; \mathrm{n}=11$ ); mixed appearances (CT grade $2 ; \mathrm{r}=0.79 ; \mathrm{n}=33$ ); and predominance of a reticular pattern (CT grade $3 ; \mathrm{r}=0.55 ; \mathrm{n}=37$ ). Functional-morphological relationships were not influenced by CT appearance. $\star$ and - - - : CT grade $1 ; \bullet$ and --- : CT grade 2 ; $\circ$ and $\longrightarrow$ : CT grade 3 .

CT appearances); and 2) patients with CT grade 1 and those with CT grades 2 and 3 as a combined group. In all analyses, functional indices were not independently related to CT pattern, after controlling for other covariates.

\section{Functional-morphological correlations and the presence of reversible disease on $C T$}

Thirty four patients underwent serial CT scanning and received prednisolone, with or without cyclophosphamide, between scans (CFA, $n=15$; FASSc, $n=19$ ). In 15

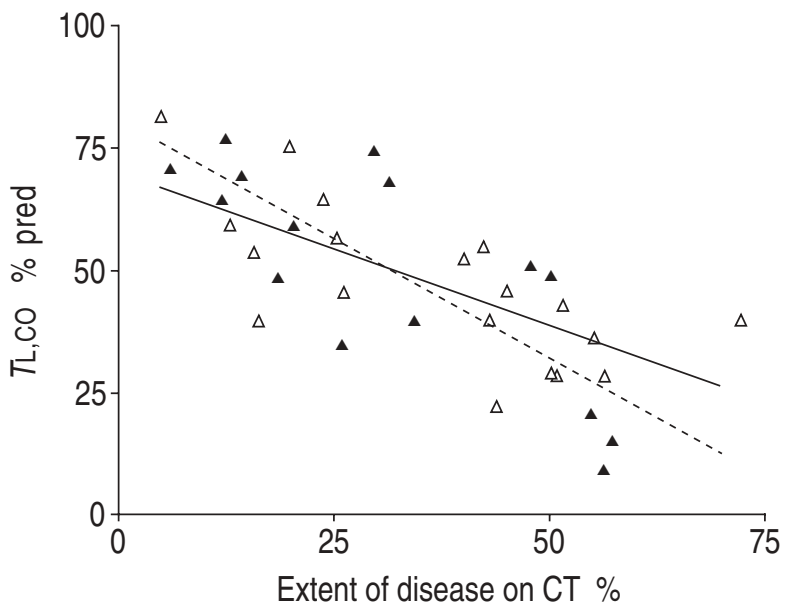

Fig. 3. - Percentage predicted TL,CO in relation to the extent of disease on CT in patients shown to have reversible disease on serial CT $(\mathrm{r}=-0.79 ; \mathrm{n}=15)$, and in patients with no regression of disease at follow-up CT $(\mathrm{r}=-0.72 ; \mathrm{n}=19)$. $\Delta$ and - - -: reversible disease; $\Delta$ and $\longrightarrow$ : irreversible disease. Functional impairment was identical in the two groups after adjustment for disease extent, smoking history, treatment at initial CT, and the type of fibrosing alveolitis (CFA versus FASSc). CFA: cryptogenic fibrosing alveolitis; FASSc: fibrosing alveolitis associated with systemic sclerosis. For further definitions see legend to figure 1 .

of the 34, regression of ground-glass attenuation was seen at follow-up CT; in 14 of these 15 instances, the overall extent of disease diminished and in one case background ground-glass attenuation resolved, but the predominant reticular pattern and overall extent of disease was unaltered. The time interval between scans did not differ significantly between patients with regression of ground-glass attenuation ( $26 \pm 13$ months) and those in whom disease was unchanged or had progressed at follow-up (18.5 \pm 11 months).

Multivariate models were constructed to examine the relationships between the severity of functional impairment and subsequent regression of disease on CT. There were no independent relationships between functional measures at initial $\mathrm{CT}$ and subsequent regression of ground-glass attenuation, after adjustment for extent of disease on CT, type of fibrosing alveolitis (CFA versus FASSc), smoking history and therapeutic status. The relationships between percentage predicted $T \mathrm{~L}, \mathrm{CO}$ and the extent of disease on CT, in patients with and without reversible disease, are shown in figure 3.

\section{Discussion}

Previous studies evaluating the functional consequences of inflammation and fibrosis in fibrosing alveolitis have been based upon histological findings at open-lung biopsy [8-11]. Discrepant findings can be ascribed to low numbers of patients studied $[8,9,11]$, the inclusion of heterogeneous groups of interstitial lung diseases [9, 11], and the non-exclusion of patients with concurrent emphysema [8, 9, 11]. Recently, CHERNIACK et al. [10] evaluated a large group of patients with CFA and found correlations between the alveolar desquamation score and TL,CO, and between interstitial cellularity and both FVC and TLC. By contrast, Fulmer et al. [8] demonstrated a relationship between oxygen desaturation 
on exercise and the degree of cell infiltration; whereas GREEN et al. [9] found interstitial inflammation to correlate poorly with functional indices. In these studies, the severity of fibrosis correlated with change in arterial oxygen tension $\left(\mathrm{Pa}_{\mathrm{a}} \mathrm{O}_{2}\right)$ on exercise, adjusted for respiratory work [8], resting $\mathrm{Pa}_{\mathrm{a}} \mathrm{O}_{2}$ [9], or bore no relationship to functional impairment [10]. CHINET et al. [11] reported relationships between the severity both of fibrosis and inflammation, and a large number of functional measures, including lung volumes, TL,CO and oxygen desaturation on exercise; however, pulmonary function tests did not indicate the relative contributions made by inflammation and fibrosis to histological disease severity.

The semiquantitative scoring of interstitial fibrosis and inflammatory cell infiltration at open lung biopsy has drawbacks, which diminish the clinical relevance of observed functional-morphological relationships. Interobserver variation in absolute grading is substantial; for many features, agreement between experienced pathologists is poor [27]. Moreover, responsiveness to treatment in fibrosing alveolitis depends upon a predominance of inflammation over fibrosis, not the absolute severity of inflammation $[1,2,28]$. There is a positive correlation between the degree of inflammatory cell infiltration and the severity of fibrosis in fibrosing alveolitis [11, 29, 30]; despite the presence of extensive inflammation in some cases, CFA patients with "mixed" histological appearances at open lung biopsy have a prognosis little different from patients with fibrotic disease [2]. Thus, correlations between functional variables and the severity of either inflammation or fibrosis in isolation have little bearing on the putative use of pulmonary function tests to distinguish between reversible and irreversible fibrosing alveolitis.

Recently, CHINET et al. [11] partially redressed this problem by showing that functional impairment does not predict whether inflammation or fibrosis makes the major contribution to overall lung damage, as judged by appearances at open-lung biopsy. However, this study did not overcome two important methodological drawbacks. Patients with CFA undergoing open-lung biopsies have less functional impairment and a better prognosis than those unfit for biopsy [31], thereby introducing a major selection bias. More importantly, biopsy material may not be representative of overall histopathological severity; the prominent regional variation seen in fibrosing alveolitis is likely to explain poor correlations between overall pathological disease severity and functional impairment in the definitive histological study of Cherniack et al. [10].

CT provides, for the first time, a sensitive means of scoring the global morphological extent of fibrosing alveolitis, and has the additional advantage of allowing all patients with fibrosing alveolitis to be studied, including those too compromised to undergo a surgical procedure. However, the use of CT to distinguish between reversible and irreversible disease has one drawback. CT appearances discriminate between patients with fibrotic disease and those with a high likelihood of reversible inflammatory disease $[6,7]$, but this distinction is not infallible. The morphological dimensions of parenchymal abnormalities may lie below scanning resolution limits, resulting in "volume averaging" and the depiction of fine intralobular fibrosis as a generalized increase in lung density, indistinguishable from the CT appearance of inflammatory cell infiltration [6, 7]; and ground-glass attenuation is more likely to represent inflammatory cell infiltration when it predominates than when it is admixed with an equally extensive reticular pattern. The inclusion of some patients with extensive fine fibrosis in the CT category of "mixed disease" has the potential to blur comparisons between inflammatory and fibrotic disease. However, our findings were unaltered when patients with "mixed disease" were excluded. Moreover, the regression of disease with treatment in patients undergoing serial CT identified a group in whom ground-glass attenuation represented inflammatory cell infiltration, and who were functionally identical to patients with irrever-sible disease.

Selection bias is an important consideration in any analysis of patients referred to a tertiary centre. There was a wide range of morphological disease extent in the present study population, but the bias towards extensive disease in CFA prompted us to include FASSc patients, referred for the evaluation of known or suspected pulmonary disease. The decision to include both types of fibrosing alveolitis in analysis was a potential source of distortion, necessitating the inclusion of the type of fibrosing alveolitis as a covariate in multivariate analysis.

In conclusion, fibrosing alveolitis patients with irreversible reticular disease were functionally identical to those with prominent ground-glass attenuation on computed tomography, after controlling for morphological disease extent. This finding persisted when serial computed tomography was used to distinguish between reversible and irreversible disease. The severity of functional impairment gives no indication of the likely nature of the underlying histopathological process in fibrosing alveolitis.

\section{References}

1. Carrington CB, Gaensler EA, Coutu RE, Fitzgerald MX, Gupta RG. Natural history and treated course of usual and desquamative interstitial pneumonia. $N$ Engl J Med 1978; 298: 801-809.

2. Turner-Warwick M, Burrows B, Johnson A. Cryptogenic fibrosing alveolitis: clinical features and their influence on survival. Thorax 1980; 35: 171-180.

3. Harrison NK, Myers R, Corrin B, et al. Structural features of interstitial lung disease in systemic sclerosis. Am Rev Respir Dis 1991; 144: 706-713.

4. Johnson MA, Kwan S, Snell NJC, Nunn AJ, Darbyshire JH, Turner-Warwick M. Randomised controlled trial comparing prednisolone alone with cyclophosphamide and low-dose prednisolone in combination in cryptogenic fibrosing alveolitis. Thorax 1989; 44: 280-288.

5. Raghu G, Depaso WJ, Cain K, et al. Azathioprine combined with prednisolone in the treatment of idiopathic pulmonary fibrosis. Am Rev Respir Dis 1991; 144: 291296.

6. Wells AU, Hansell DM, Rubens MB, Cullinan P, Black $\mathrm{CM}$, du Bois RM. The predictive value of appearances on thin-section computed tomography in fibrosing alveolitis. Am Rev Respir Dis 1993; 148: 1076-1082.

7. Wells AU, Rubens MB, du Bois RM, Hansell DM. Serial $\mathrm{CT}$ in fibrosing alveolitis: prognostic significance of the initial pattern. Am J Radiol 1993; 161: 1159-1165. 
8. Fulmer JD, Roberts WC, von Gal ER, Crystal RG. Morphologic-physiologic correlates of the severity of fibrosis and degree of cellularity in idiopathic pulmonary fibrosis. J Clin Invest 1979; 63: 665-676.

9. Green GM, Graham WGB, Hanson JS, et al. Correlated studies of interstitial pulmonary disease. Chest 1976; 69 (Suppl.): 263-284.

10. Cherniack RM, Colby TV, Flint A, et al. Correlation of structure and function in idiopathic pulmonary fibrosis. Am J Respir Crit Care Med 1995; 151: 1180-1188.

11. Chinet T, Jaubert F, Dusser D, Danel C, Chretien J, Huchon GJ. Effects of inflammation and fibrosis on pulmonary function in diffuse lung fibrosis. Thorax 1990; 45: 675-678.

12. Collins CD, Wells AU, Morgan RA, et al. Observer variation in pattern type and extent of disease in fibrosing alveolitis on thin section computed tomography. Clin Radiol 1994; 49: 236-240.

13. Mathieson JR, Mayo JR, Staples CA, Müller NL. Chronic diffuse infiltrative lung disease: comparison of diagnostic accuracy of CT and chest radiography. Radiology 1989; 171: 111-116.

14. Tung KT, Wells AU, Rubens MB, Kirk JME, du Bois RM, Hansell DM. Accuracy of typical computed tomography appearances of fibrosing alveolitis. Thorax 1993 48: 334-338.

15. Subcommittee for Scleroderma Criteria of the American Rheumatism Association Diagnostic and Therapeutic Criteria Committee. Preliminary criteria for the classification of systemic sclerosis (scleroderma). Arthritis Rheum 1980; 23: 581-590.

16. Müller NL, Staples CA, Miller RR, Vedal S, Thurlbeck WM, Ostrow DN. Disease activity in idiopathic pulmonary fibrosis: CT and pathologic correlation. Radiology 1987; 165: 731-734.

17. Wells AU, Hansell DM, Corrin B, et al. High resolution computed tomography as a predictor of lung histology in systemic sclerosis. Thorax 1992; 47: 738-742.

18. Müller NL, Miller RR, Webb WR, Evans KG, Ostrow DN. Fibrosing alveolitis: CT-pathologic correlation. Radiology 1986; 160: 585-588.

19. Nishimura K, Kitaichi M, Izumi T, Nagai S, Kanaoka $\mathrm{M}$, Itoh H. Usual interstitial pneumonia: histologic correlation with high resolution CT. Radiology 1992; 182: 337-342.

20. Quanjer PH. Standardised lung function testing. Clin Respir Physiol 1983; 19 (Suppl.) 5: 1-95.

21. Riley RL, Cournand A. Ideal alveolar air and the analysis of ventilation-perfusion relationships in the lungs. $J$ Appl Physiol 1949; 1: 825-847.

22. Watters LC, King TE, Schwarz MI, Waldron JA, Stanford RE, Cherniack RM. A clinical, radiographic, and physiologic scoring system for the longitudinal assessment of patients with idiopathic pulmonary fibrosis. Am Rev Respir Dis 1986; 133: 97-103.

23. Chinn S. Statistics in respiratory medicine: repeatability and method comparison. Thorax 1991; 46: 454-456.

24. Fleiss JL. Statistical methods for rates and proportions. New York, NY, Wiley, 1973.

25. Coblentz CL, Babcook CJ, Alton D, Riley BJ, Norman G. Observer variation in detecting the radiologic features associated with bronchiolitis. Invest Radiol 1991; 26: 115-118.

26. Kleinbaum DG, Kupper LL, Müller R. Applied regression analysis and other multivariate methods. Boston, MA, Kent, 1988.

27. Cherniack RM, Colby TV, Flint A, et al. and the BAL Co-operative Group Steering Committee. Quantitative assessment of lung pathology in idiopathic pulmonary fibrosis. Am Rev Respir Dis 1991; 144: 892-900.

28. Wright PH, Heard BE, Steel SJ, Turner-Warwick M. Cryptogenic fibrosing alveolitis: assessment by graded trephine lung biopsy histology compared with clinical, radiographic, and physiological features. Br J Dis Chest 1981; 75: 61-70.

29. Haslam PL, Turton CWG, Heard B, et al. Bronchoalveolar lavage in pulmonary fibrosis: comparison of cells obtained with lung biopsy and clinical features. Thorax 1980; 35: 9-18.

30. Wells AU, Lorimer S, Majumdar S, et al. Fibrosing alveolitis in systemic sclerosis: increase in memory Tcells in lung interstitium. Eur Respir J 1995; 8: 266-271.

31. Wells AU, Cullinan P, Hansell DM, et al. Fibrosing alveolitis associated with systemic sclerosis has a better prognosis than lone cryptogenic fibrosing alveolitis. Am J Respir Crit Care Med 1994; 149: 1583-1590. 